\title{
Differential gene expression during colon-to-lung metastasis
}

\author{
SUNG-HYUN KIM ${ }^{1 *}$, SO-JUNG CHOI ${ }^{1 *}$, YONG BEOM CHO ${ }^{2}$, MIN-WOONG KANG ${ }^{3}$, \\ JINSEON LEE ${ }^{1}$, WOO-YONG LEE ${ }^{2}$, HO KYUNG CHUN ${ }^{2}$, YONG SOO $\mathrm{CHOI}^{3}$, \\ HONG KWAN KIM ${ }^{3}$, JUNGHO HAN ${ }^{4}$ and JHINGOOK KIM ${ }^{1,3}$ \\ ${ }^{1}$ Cancer Research Center, Center for Clinical Research, Samsung Biomedical Research Institute; \\ Departments of ${ }^{2}$ Surgery, ${ }^{3}$ Thoracic Surgery and ${ }^{4}$ Pathology, Samsung Medical Center, \\ Sungkyunkwan University School of Medicine, Seoul 135-710, Republic of Korea
}

Received July 21, 2010; Accepted October 27, 2010

DOI: $10.3892 /$ or.2011.1142

\begin{abstract}
Primary tumors in certain metastatic cases have potential dissemination mechanisms. However, they often lack the potential to colonize distant microenvironments, and consequently the disseminated cancer cells enter into a state of latency which can last for years. In order to investigate the metastatic colonization potential at the gene expression level, we compared such primary tumors with their matching, actively proliferating metastatic tumors. Six pairs of colonto-lung metachronous tumor samples were examined for the expression levels of 84 well-known metastatic genes using the quantitative RT-PCR-based PCR Array technology. The unsupervised hierarchical clustering of all 12 samples together, resulted in the formation of one closely related cluster by the primary tumors, but highly diversified ones by the metastatic tumors. A pair-wise comparison of the matching primarymetastatic tumors showed that different groups of genes were activated in the lung metastases. Therefore we charted specific genes involved in the genetic diversification processes. A number of these genes showed similar differential expression $(\Delta \mathrm{Ct})$ patterns in all the patients. These were the cancer cell-, the microenvironment- and the stem cell-specific gene groups. In conclusion, the results suggest that the primary colorectal cancer cells are diversified as regards colonization of the lung, which could explain why the effective therapies for primary colorectal cancers are often not appropriate for controling the growth of pulmonary metastases.
\end{abstract}

\section{Introduction}

Metastasis can be viewed as two consecutive cellular processes: Dissemination of the cancer cells from the primary tumor to distant sites, and colonization of the disseminated

Correspondence to: Dr Jhingook Kim, Cancer Research Center, Center for Clinical Research, Samsung Biomedical Research Institute, 50 Ilwon-Dong, Kangnam-Ku, Seoul 135-710, Republic of Korea E-mail: jkimsmc@skku.edu

${ }^{*}$ Contributed equally

Key words: colorectal cancer, lung cancer, metastasis, colonization, latency cancer cells of microscopic size into full-blown metastatic malignancies $(1,2)$. When disseminated to a distant microenvironment, most cancer cells can no longer grow in the alien microenvironment, and thus enter into a state of latency which can last for months or years until they discover new growth mechanisms (3-5). Understanding how the micrometastases come out of their latent state to colonize the distant organ is of prime interest, as it could lead us to a therapeutic means of controling the process. However, such studies are generally not practically feasible, as metastasectomies are rarely performed, and even in those rare cases, the invasiveness of the matching primary tumors often decreases during the latent years.

However, recent trends of aggressive metastasectomy, particularly of lung metastases of colorectal origin $(6,7)$, could shed a light on this problem. Surgical resection of metastatic tumors is usually performed when, i) recurrence is not detected in the primary site, ii) complete surgical removal of the metastases is feasible, iii) there is no evidence of metastasis in other organs other than the lung, and iv) the patient can tolerate the surgery (6). The tumors removed thereby, are chronologically separated during the latent periods, and genetic differences $(5,8)$ reflect the changes in their ability to colonize the lung.

Each pair of primary colon cancers with lung metastases from the same patients, are good study models as they have identical germ-line genetics and common cancer lineages (5-10), with limited genetic changes associated with the colonization. In this study, we investigated the changes in gene expression of known metastatic-related genes (11) [SABiosciences human tumor metastasis (PAHS-028) finder $\mathrm{RT}^{2}$ profiler PCR array and the RT2 SYBR-Green/ROX PCR Master Mix (APMM012C and PA-012-24, SABiosciences)] in colon-to-lung metachronous tumors samples, and charted them according to regulatory pathways. Consistent with the diverse cell-cell interactions in the lung implicated in the colonization mechanisms (12-14), our results demonstrate diversification during colon-to-lung metastasis.

\section{Materials and methods}

Patient and sample selection. Six pairs of primary colon tumor tissues and their matching lung metastases (Table I) 
Table I. Summary of the clinical information.

\begin{tabular}{|c|c|c|c|c|c|c|c|}
\hline \multirow[b]{2}{*}{ Case no. } & \multirow[b]{2}{*}{ Gender } & \multirow[b]{2}{*}{ Age (years) } & \multicolumn{3}{|c|}{ Primary tumors } & \multicolumn{2}{|c|}{ Metastatic tumors } \\
\hline & & & Size $(\mathrm{cm})$ & apStage & Primary site & Metastatic site & ${ }^{\mathrm{b}}$ Latent interval (Mo) \\
\hline 1 & $\mathrm{~F}$ & 36 & 4.8 & IIIC & Lower rectum & Lung-left & 18 \\
\hline 2 & $\mathrm{~F}$ & 49 & 4.4 & IIIA & Lower rectum & Lung-bilateral & 24 \\
\hline 3 & $\mathrm{~F}$ & 57 & 5.2 & IIIB & Upper rectum & Lung-left & 22 \\
\hline 4 & M & 65 & 3.5 & I & Lower rectum & Lung-bilateral & 34 \\
\hline 5 & M & 70 & 3.5 & IIA & Upper rectum & Lung-bilateral & 36 \\
\hline 6 & $\mathrm{~F}$ & 53 & 5.2 & IIIC & S-colon & Lung-left & 24 \\
\hline
\end{tabular}

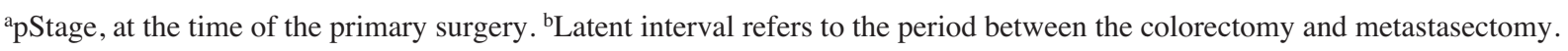

were collected after surgical resections which were carried out in Samsung Medical Center between 2001 and 2008. We followed the protocols approved by the institutional review board with prior consent given from all patients. Necrotic regions were avoided and the tumor masses were snap-frozen and maintained in liquid nitrogen before RNA extraction. Prior to the RNA preparations, the medical records and hematoxylin/eosin-stained slides of the specimens were reviewed to remove the necrotic tissues and the intervening normal tissues, thus abiding by the World Health Organization histopathological criteria. Specimens with tumor cell contents of $>90 \%$ were selected and processed for further analysis.

Gene expression profiling by $R T^{2}$ Profiler $^{\mathrm{TM}}$ PCR Array. Total RNAs were extracted from the frozen lung metastatic tumors and their respective controls, as well as from the colon primary tumors, using the NucleoSpin RNA kit, and cDNA was synthesized using the $\mathrm{RT}^{2}$ First Strand Kit (SABiosciences, cat. no. C-03). The human tumor metastasis (PAHS-028) finder RT2 profiler PCR array and the RT2 SYBR-Green/ROX PCR Master Mix (APMM012C and PA012-24, SABiosciences) were used to quantitatively analyze the gene expression levels of 84 known metastatic genes and 5 house-keeping genes, following the instructions of the manufacturer. The array analysis was carried out according to the manufacturer's instructions. The negative controls included by the manufacturer in 96-well format, generated data that ensured the lack of contamination. PCR was performed using the ABI 7300 Real-Time PCR System (Applied Biosystems). In order to confirm the plate to plate consistency, duplicate and triplicate reactions were carried out.

Data analyses. $\mathrm{Ct}$ values were normalized to the deviations against the average of 5 housekeeping genes: B2M, RPRT1, $H P R T 1, G A P D H$ and ACTB. The differential gene expression was estimated as, $\Delta \mathrm{Ct}=\mathrm{Ct}_{\text {(lung metastases) }}-\mathrm{Ct}_{\text {(colon primary) }}$, and fold change $=2^{(-\Delta \mathrm{Ct})}$. Quantitative analysis of the array data on the primary colon and the metastatic lung tumors was carried out by the Web-Based PCR Array Data Analysis software provided by the manufacturer (http://www.sabiosciences.com). In order to examine the relative expression levels among the 6 cases, $-\mathrm{Ct}_{\text {(lung metastases) }}$ and $-\mathrm{Ct}_{\text {(colon primary) }}$ values were used as absolute expression levels. Fold changes of $>2$-fold were considered to be significant.

\section{Results}

Analyses of gene expression $(C t)$. In order to examine the changes in gene expression profiles which occurred between the primary and metastatic tumors, quantitative RT-PCR was carried out for 84 genes using the human tumor metastasis finder $\mathrm{RT}^{2}$ profiler PCR array. Consistent with previous studies using RT-PCR, Ct values were highly reproducible in assayable genes with a relatively wide range of expression levels among the samples (9): The correlation between the duplicates was $>0.95$ in the highly assayable genes, and the average correlation was 0.92 . When the gene expression was relatively constant at low levels, the small fluctuation was frequently not reproduced, and the correlation decreased as low as 0.82 . For an overview of the gene expression patterns across the samples, unsupervised two-dimensional hierarchical clustering was carried out (Fig. 1) with $\mathrm{Ct}$ values. Most of the genes showed unique expression patterns, although 26 out of the 84 genes clustered into 2 closely related groups with similar expression patterns (marked by a blue square: A large cluster of 21 genes (including ITGB3, ITGA7, TGF- $\beta 1$, TNFSF10, APC, MCAM, IGF 1, COL4A2, HGF, CST7, CDH6, MMP2, TIMP2, CD44, CD82, PTEN, GNRH1, $M T S S 1$ and $S M A D 2)$, and a small cluster of 3 genes (VEGFa, $I L 8 R B$ and $S R C)$. The similarity in their gene expression patterns was estimated by correlation: CDH6-CST7 (0.94), SMAD2-CXCL12 (0.94), CXCL12-CST7 (0.92), PTENMTSS1 (0.92), and 11 pairs showed a correlation of $>0.82$ in the large cluster, and $0.81-0.88$ in the small cluster. These similarities are comparable to those of single gene duplicates. These data raise the possibility that the expression patterns of these genes could be regulated by a common upstream regulatory mechanism.

Gene expression $(C t)$ patterns in the primary vs. metastatic tumors. As shown in the clustergram in Fig. 1B, all 6 primary tumors (4-P, 1-P, 6-P, 2-P, 3-P and 5-P) clustered into 1 closely related group, with short phylogenetic distances, as marked by the short length of the vertical lines of the tree. 


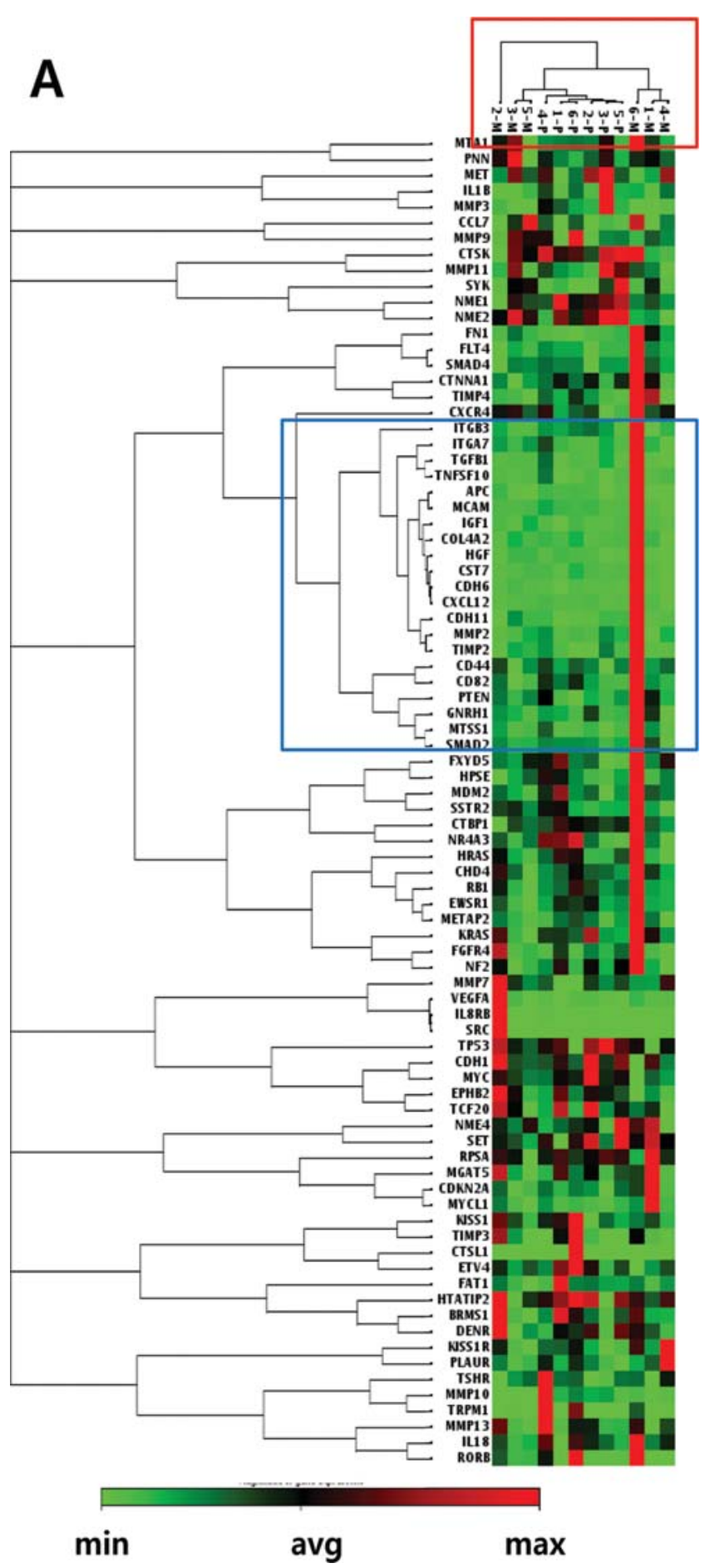

Particularly, the primary tumor of case no. 3 was highly related to case no. 5 (3-P and 5-P) and to a lesser extent to case no. 2. The primary tumor of case no. 1 was also highly related to the primary tumor of case no. 6 (1-P and 6-P) and again to a lesser extent to case no. 2 . These relationships among the cases were also conserved among the metastatic tumors. Case nos. 2, 3 and 5 and 6, 1 and 4, were closely related to one another. The results indicate that similar molecular pathways were activated in the primary tumors compared to the metastatic tumors. On the contrary, the metastatic tumors formed relatively remote groups with only 1,2 , or 3 samples per group: The metastatic tumor of case
B

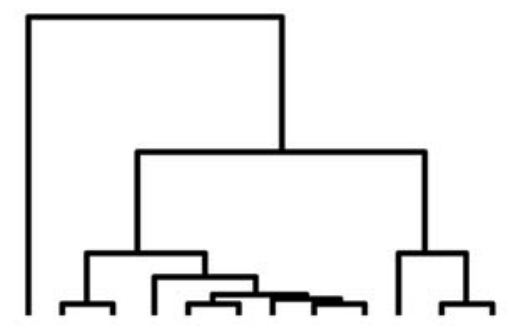

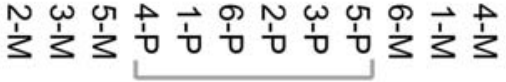

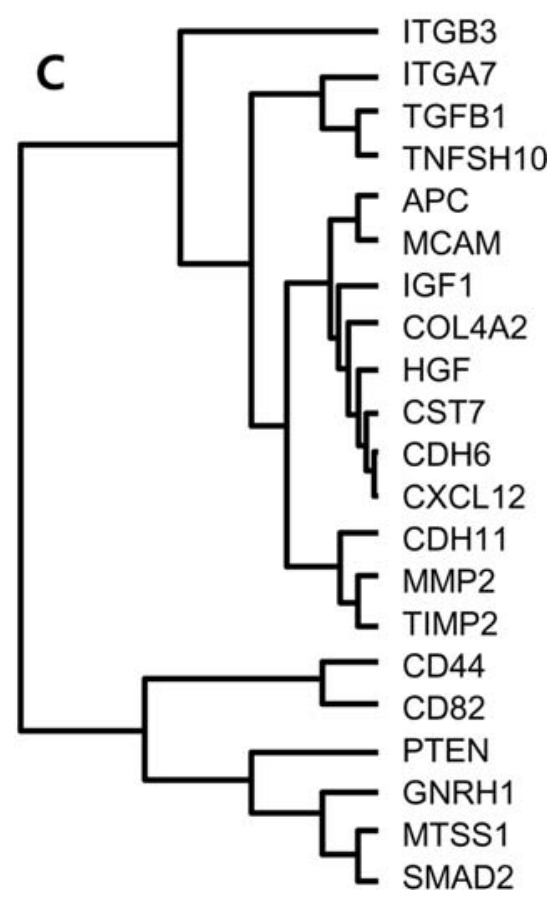

Figure 1. (A) Unsupervised two-dimensional hierarchical clustering. Red square is the cluster of the tumors magnified in (B), and blue square is the cluster of genes magnified in (C). (B) Cluster of the tumors. All the 6 primary colon tumors were relatively similar to one another (bracket), but the metastatic lung tumors were highly diverged from one another and from the primary tumors. (C) Cluster of closely related genes.

no. 2 (2-M) was most remotely related to the other samples, the metastatic tumors of case nos. 3 and 5 (3-M and 5-M) were relatively related to the primary tumor group, and the metastatic tumor of case no. $6(6-\mathrm{M})$ was related to another group formed by the metastatic tumors of case nos. 1 and 4 . Taken together, these data suggest that the 6 patients had similar gene expression profiles in the primary tumors, which became diversified in the metastatic tumors.

Differentially up-regulated genes. In order to investigate the upstream gene regulation, differential gene expression $(\Delta \mathrm{Ct})$ was calculated for individual genes in each patient. In order 


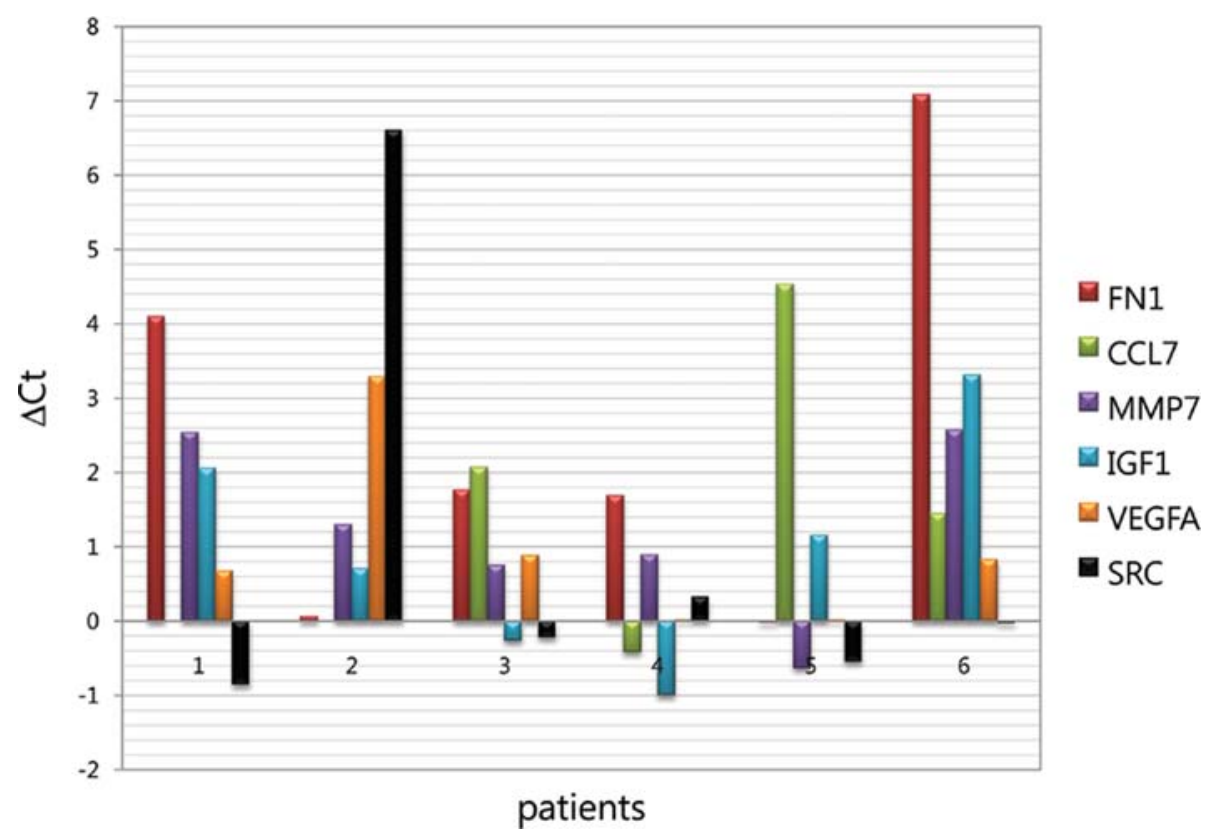

Figure 2. Expression of top 6 up-regulated genes in the metastatic tumors. Positive y axis, up-regulation; negative y axis, down-regulation in the pulmonary metastases. Each patient expressed different batteries of genes demonstrating genetic diversity among the metastatic tumors in the lung.

Table II. The genes up-regulated in the lung metastases.

\begin{tabular}{|c|c|c|c|}
\hline $\mathrm{No}^{\mathrm{a}}$ & Gene & No. & Gene \\
\hline 1 & $F N$ & 22 & MTAl \\
\hline 2 & $C C L 7$ & 23 & $S Y K$ \\
\hline 3 & $M M P 7$ & 24 & $P N N$ \\
\hline 4 & $I G F 1$ & 25 & GNRHI \\
\hline 5 & $V E G F \alpha$ & 26 & PLAUR \\
\hline 6 & $S R C$ & 27 & $C D 44$ \\
\hline 7 & CXCR4 & 28 & KISSIR \\
\hline 8 & CDH6 & 29 & FGFR4 \\
\hline 9 & MTSS1 & 30 & $C D K N 2 A$ \\
\hline 10 & CDHII & 31 & NME4 \\
\hline 11 & TNFSF 10 & 32 & $T G F \beta 1$ \\
\hline 12 & MYCL1 & 33 & $A P C$ \\
\hline 13 & PTEN & 34 & SMAD2 \\
\hline 14 & ITGA7 & 35 & SSTR2 \\
\hline 15 & FXYD5 & 36 & CHD4 \\
\hline 16 & FLT4 & 37 & CXCL12 \\
\hline 17 & $H G F$ & 38 & CST7 \\
\hline 18 & $S M A D 4$ & 39 & IL18 \\
\hline 19 & $R O R B$ & 40 & MMP9 \\
\hline 20 & COLAA2 & 41 & MGAT5 \\
\hline 21 & TIMP2 & 42 & $M D M 2$ \\
\hline
\end{tabular}

${ }^{a}$ The list is in the decreasing order of fold change in expression levels, with the most up-regulated gene (by fold change) in the lung metastases at the top of the list. to identify the mechanisms that are newly activated in the metastatic tumors, the up-regulated genes in the metastatic lung tumors were listed (Table II) in the order of the sum total of $\Delta \mathrm{Ct}$. As a visual sample of the gene activities in each patient, the differential expression of the top 6 genes in the list were plotted (Fig. 2). Each one of the patients showed a unique combination of activated genes: FN1, MMP7 and $I G F 1$ in patient 1 , only counting $\Delta \mathrm{Ct}>2$ as significant upregulation, VEGFA and $S R C$ in patient 2, only $C C L 7$ in patient 3, no significant up-regulation of the 6 genes in patient 4, CCL7 in patient 5, and FN1, MMP7 and IGF1 in patient 6 . Not only were the gene combinations unique, but also the expression levels of each gene ranged up to $7 \Delta \mathrm{Ct}$ (or $>100$ fold changes). These data demonstrate how the diversification of the metastatic tumors was achieved at the gene expression level.

Pathway analysis of the differentially expressed genes. As similarities were observed in the gene expression $(\mathrm{Ct})$ patterns (shown in Fig. 1), the similarities of the $\Delta \mathrm{Ct}$ patterns across the patients were examined by hierarchical clustering (Fig. 3A). Consistent with the data in Fig. 1, the 6 tumors did not form closely related clusters except for cases 1 and 3 . However, 21 genes formed 3 related groups: Group no. 1 included IGF, TIMP2, CXCR4, TGFß1, MMP9, PTEN and MTSS1. Group no. 2 included CX3CL12, CDH11, COLAA2, CST7, CDH6, SMAD4, GNRH1, FLT4 and TNFSF10. Group no. 3 included CHD4, CD44, MTA1, MGAT5, and PNN. Among the 3 groups, group no. 1 showed the longest phylogenetic distance, as marked by the length of the horizontal lines of the phylogenetic tree (or the least similarity in the regulatory patterns), and group no. 3 the shortest, among the group members. The differential expression patterns of these 3 groups were further analyzed by scatter plots (Fig. 3B-D). 
A.

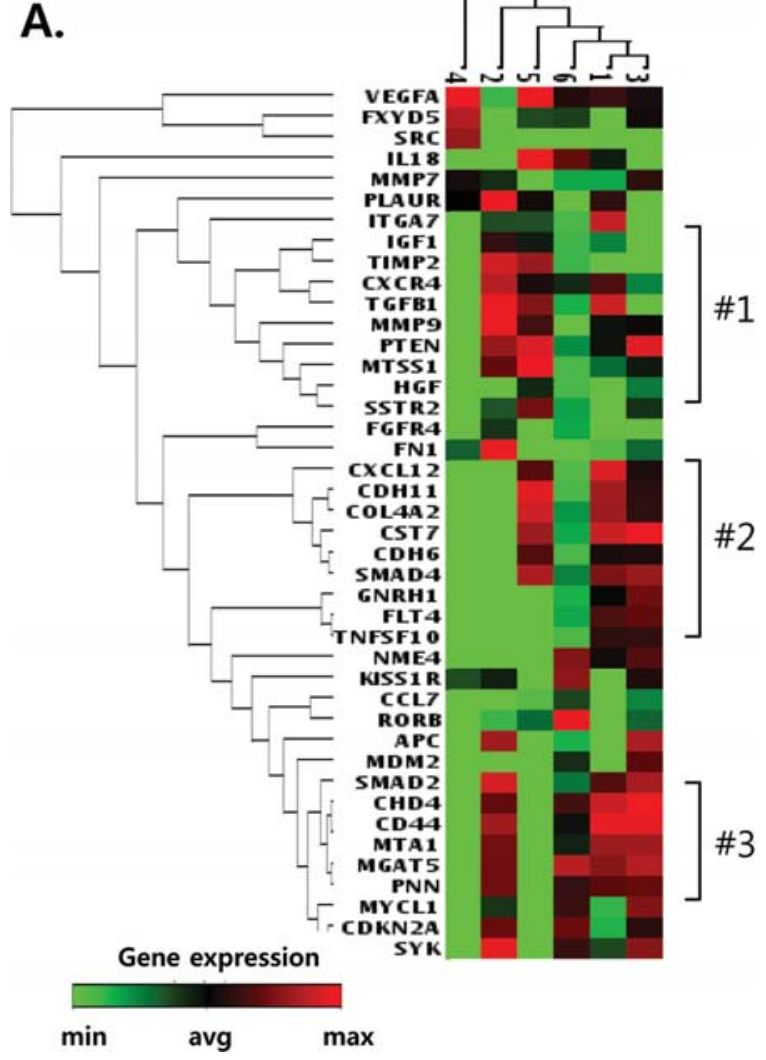

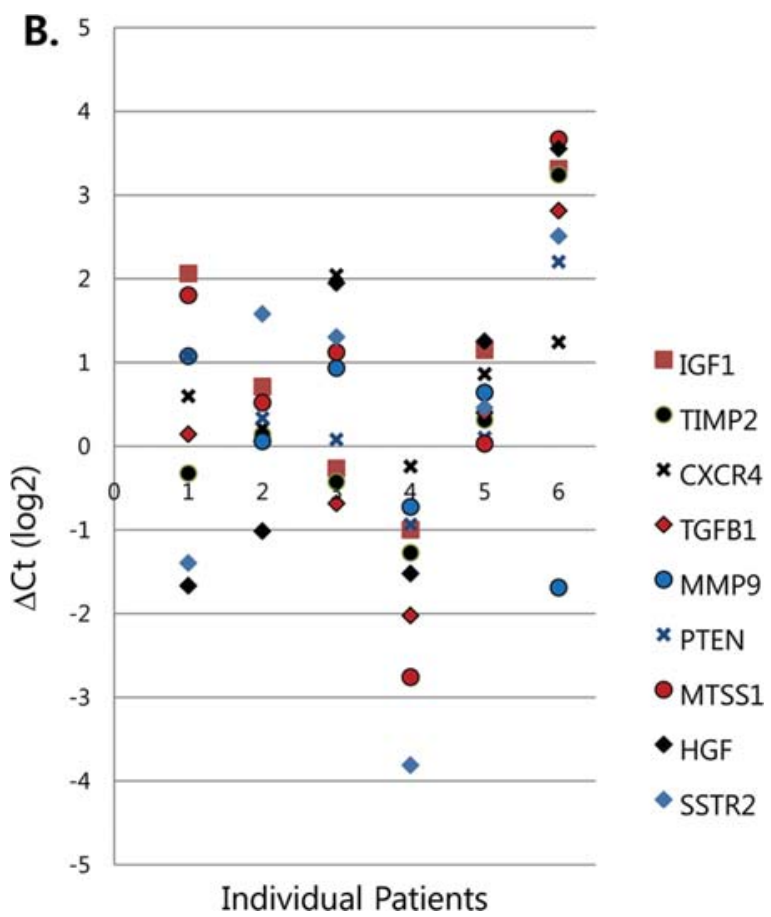

D.

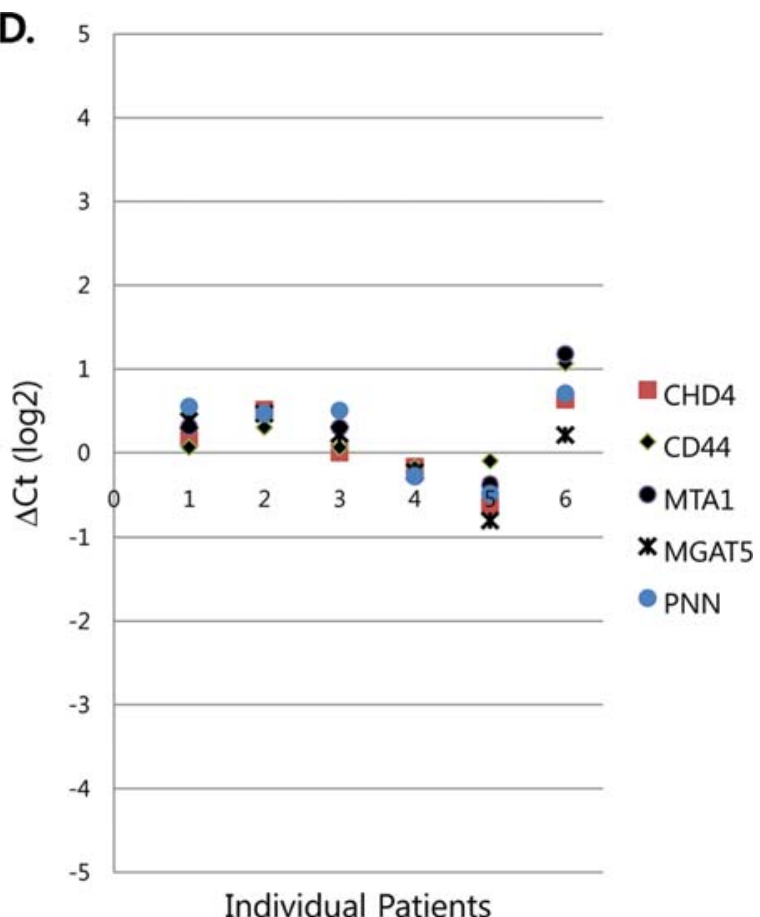

Figure 3. Gene clusters with similar differential gene expression patterns. (A) Hierarchical clustering of $\Delta \mathrm{Ct}$ showed that the majority of the up-regulated genes formed 3 groups with closely-related patterns. (B) Scatter plots of the cancer cell-specific gene groups. (C) Scatter plots of the lung microenvironmentspecific gene groups. Patient no. 5 had expressions clustered around the zero $\Delta \mathrm{Ct}$ value, and consequently was differentially color coded as either up- or down-regulated. Therefore, the color coding in patient no. 5 was ignored in gene grouping. (D) Scatter plots of the stem cell-specific gene groups. These data suggest that the activity of a number of genes involved in metastasis could be regulated by a limited number of upstream regulatory mechanisms.

We discovered that patient no. 5 had gene expressions clustered around the zero $\Delta \mathrm{Ct}$ value, and was consequently differentially color coded as either up- or down-regulated.
Therefore, the color coding in patient no. 5 was ignored in gene grouping. These data show that the majority of genes clustered into 3 groups with similar regulatory $(\Delta \mathrm{Ct})$ patterns. 


\begin{tabular}{|c|c|c|c|}
\hline & $\underline{\text { Recurrence state }}$ & Comparison & $\Delta$ Potentials \\
\hline Type 1 & $R$ & between patients & dissemination \\
\hline Type 2 & $M$ & between events & colonization \\
\hline
\end{tabular}

Figure 4. Metastatic study models: Depending on the metastatic potentials of the surgical samples, study models can be divided into two types: Type 1, the transverse model for the dissemination that compares the primary tumors that recur later, and those that do not recur, and type 2 , the longitudinal model for the colonization that compares the tumor samples from the same patients before and after colonization (identical genetic backgrounds). P, primary tumors; M, metastatic tumors; R, recurrence; NR, non-recurrence.

\section{Discussion}

Study models for metastasis can be divided into 2 types based on the genetic potentials of the specimens used (Fig. 4). Type 1 studies have compared the non-metastatic to metastatic primary tumors of different patients to discover genetic markers for metastasis $(15,16)$. However, more specifically, they were designed to study dissemination, as the focused clinical variable is the presence or absence of metastatic spreads at the time of surgical resection. By the nature of the study design, the samples of type 1 cannot be obtained from the same patient, and therefore type 1 study models often include many samples to overcome the person-to-person genetic variation $(15,16)$. On the contrary, type 2 studies (longitudinal study models) have compared the 2 chronological samples separated by the colonization event under the identical genetic background of the same patient. Unfortunately, type 2 samples are hard to come by, as only a small fraction of cases suffice the conditions for metastasectomy, and relatively long follow-up periods are required, which is inversely proportional with the RNA quality. Nonetheless, more of these samples are expected to be available in the future due to recent trends towards aggressive metastasectomy $(6,7)$, and therefore, the careful preservation of these samples could be of prime importance for cancer biologists to study metastatic colonization.

It is important to select primary tumors with well defined latency (Table I), in order to rule out the possibility of primary tumors already equipped with colonization potentials. Though latency is defined as the period between the arrival of disseminated cancer cells and the onset of log-phase growth, it is technically challenging to measure it, mainly due to the fact that it is unclear when dissemination occurs. Twelve months of median survival time of the metastatic cases (17) is the growth phase from the detection of metastatic tumors to death, and therefore, the growth phase prior to the formation of detectable size could occur within months, not years. However, the dissemination can occur at anytime during the decades of evolution in the primary site. If it occurs at relatively late stages, early diagnosis can prevent metastasis by surgical resection before the dissemination. Studies on early diagnostic efforts have demonstrated $20 \%$ reduction in metastatic mortality (18), which conversely suggests that the remaining majority of metastatic cases cannot be prevented by early diagnosis, or that dissemination occurrs even before the early diagnostic tests. Therefore, the actual latency must be significantly longer than the latent periods between the colorectomy and metastasectomy.

In order to chart the genes associated with lung colonization in these patients, we examined how the expression of known metastatic-related genes changes from the primary to the metastatic tumors by quantitative RT-PCR. When the data were analyzed by unsupervised hierarchical clustering, despite the diverse stages of the primary tumors (stages I, II and III) as determined at the time of the colorectomy, their gene expression patterns turned out to be relatively similar to one another (Fig. 1A). Despite the diverse genetic alterations accumulated in the primary colorectal tumors (9), these data suggest that the dissemination mechanism could be part of the conserved patterns. On the contrary, the gene expression patterns were found to be highly diversified after colonization in the lung metastases, suggesting that diverse growth mechanisms are feasible.

The genes up-regulated during colonization are charted in Table II. These were grouped into 3 related clusters based on the similarities in the $\Delta \mathrm{Ct}$ patterns (Fig. 3). When their reported functions were surveyed, no pathway distinction could be made between the groups, reflecting the cross-talks between various functional pathways $(19,20)$. The pathway analyses were further confounded by cellular context, as a number of genes were expressed in various cell types where they carried out distinct genetic traits. For example, TGF- $\beta$ is expressed in myeloid, mesenchymal and in cancer cells themselves (21), and depending on the cellular context in which it is activated, it can act either as a tumor suppressor, or an oncogene $(22,23)$. Therefore, the grouping of genes could be meaningful if the cell types could be identified, and if there are 'seed' or 'soil' factors involved (3).

The reported functions of the 3 groups of genes reflected the interactions among various cell types: The colorectal cancer cells, the lung microenvironment, and the cancer stem cells (12-14). Specifically, group 1 was named as cancer cell factors, as it contains genes such as CXCR4 (24), PTEN (25), MTSS1 $(26,27)$ and SSTR2 $(28)$ that are known to be expressed and function within the tumor cells. Groups 2 and 3, were named as the lung 'soil' factors, as they contain genes expressed at high levels in the lung microenvironments, such as COL4A2, CXCL12, CDH11, CST7, FLT4 and TNFSF10 (29). Among them, CXCL12 is a well-known 'soil' factor expressed in high levels in the injured tissues to attract specialized cells for wound healing, or in the metastatic sites, to attract the disseminated cancer cells $(30,31)$. CXCL12 
binds to $C X C R 4$ in the disseminated cancer cells, which triggers intracellular signaling for cell proliferation (32). FLT4 is another 'soil' factor required by the stromal cells for angiogenesis (33). These studies indicate that both of the regulatory clusters, one for the lung soil factors and the other one for the cancer cell factors, could be necessary for the colonization of the lung by colorectal cancer cells. Group 4 was named as the stem cell factors (34-37), as it contains the genes with known functions in stem cell activities: $C D 44$ is one of the best known stem cell markers (38), and MTAl is known to form a transcriptional repression complex in which it directly interacts with OCT4 and Nanog to maintain stemness (39). CHD4 has been reported to function during regeneration and wound healing (40). Targeted disruption of MGAT5 caused depletion of adult stem cells $(41,42)$. PNN is the only member of the stem cell factor group that has not been reported to function in stem cells. Though the $\Delta \mathrm{Cts}$ of the stem cell factors are not prominently up-, or downregulated in the metastatic tumors, this could be due to the scarcity of the stem cells in the high background expression. Evidently, not all the genes in each group have been reported to have postulated functions, which could be due to technical problems or to a study design with a limited number of samples. Validation studies with an expanded number of samples are required in order to confirm the regulatory groups and their constituents.

In this study, we used the primary-to-metastatic tumor pairs of the same patients as a model to study metastatic colonization. The genes that are differentially activated during the colonization of the lung by colorectal cancer cells were charted from 84 well-known metastatic genes, and the regulatory pathways were discussed. When the expression profiles were compared, all the primary tumors formed one cluster with similar gene expression, which became highly diversified in the lung metastases. These data suggest that there could be multiple colonization mechanisms. Further studies are required in order to validate the clinical significance of the findings.

\section{Acknowledgements}

This study was supported by Samsung Biomedical Research Institute grant nos. SBRI C-A6-411 and C-A7-802, and the National Research Foundation of Korea (NRF) grants funded by the Korean government (MEST) (nos. R01-2006-00011114-0 and NTX2091113).

\section{References}

1. Nguyen DX, Paula DB and Massagué J: Metastasis: from dissemination to organ-specific colonization Nat Rev Cancer 9: 274-284, 2009

2. Fidler IJ: The pathogenesis of cancer metastasis: the 'seed and soil' hypothesis revisited. Nat Rev Cancer 3: 453-458, 2003

3. Kjeldsen BJ, Kronborg O, Fenger C and Jørgensen OD: The pattern of recurrent colorectal cancer in a prospective randomized study and the characteristics of diagnostic tests. Int J Colorectal Dis 12: 329-334, 1997

4. Weigelt B, Glas AM, Wessels LF, Witteveen AT, Peterse JL and van't Veer LJ: Gene expression profiles of primary breast tumors maintained in distant metastases. Proc Natl Acad Sci USA 100: 15901-15905, 2003.

5. Shah SP, Morin RD, Khattra J, et al: Mutational evolution in a lobular breast tumour profiled at single nucleotide resolution. Nature 461: 809-813, 2009.
6. Park JS, Kim HK, Choi YS, Kim K, Shim YM, Jo J, Lee WY, Chun HK, Park YS, Kang WK and Kim J: Outcomes after repeated resection for recurrent pulmonary metastases from colorectal cancer. Ann Oncol 21: 1285-1289, 2010.

7. Riquet M, Foucault C, Cazes A, et al: Pulmonary resection for metastases of colorectal adenocarcinoma. Ann Thorac Surg 89: 375-380, 2010.

8. Fidler IJ and Kripke ML: Genomic analysis of primary tumors does not address the prevalence of metastatic cells in the population. Nat Genet 34: 23, 2003

9. Jones S, Chen WD, Parmigiani G, et al: Comparative lesion sequencing provides insights into tumor evolution. Proc Natl Acad Sci USA 105: 4283-4288, 2008.

10. Schmidt-Kittler O, Ragg T, Daskalakis A, et al: From latent disseminated cells to overt metastasis: genetic analysis of systemic breast cancer progression. Proc Natl Acad Sci USA 100: 7737-7742, 2003.

11. Wang J, Shiozawa Y, Wang J, et al: The role of CXCR7/RDC1 as a chemokine receptor for CXCL12/SDF-1 in prostate cancer. J Biol Chem 283: 4283-4294, 2008.

12. Psaila B and Lyden D: The metastatic niche: adapting the foreign soil. Nat Rev Cancer 9: 285-293, 2009.

13. Polyak K and Weinberg RA: Transitions between epithelial and mesenchymal states: acquisition of malignant and stem cell traits. Nat Rev Cancer 9: 265-273, 2009.

14. Joyce JA and Pollard JW: Microenvironmental regulation of metastasis. Nat Rev Cancer 9: 239-252, 2009.

15. Lee ES, Son DS, Kim SH, et al: Prediction of recurrence-free survival in postoperative non-small cell lung cancer patients by using an integrated model of clinical information and gene expression. Clin Cancer Res 14: 7397-7404, 2008.

16. Potti A, Mukherjee S, Petersen R, et al: A genomic strategy to refine prognosis in early-stage non-small-cell lung cancer. $\mathrm{N}$ Engl J Med 355: 570-580, 2006.

17. Omura K: Advances in chemotherapy against advanced or metastatic colorectal cancer. Digestion 77 (Suppl 1): 13-22, 2008.

18. La Vacchia C: Fecal occult blood screening for colorectal cancer: open issues. Ann Oncol 13: 31-34, 2002

19. Cheung P and Dennis JW: Mgat5 and Pten interact to regulate cell growth and polarity. Glycobiology 17: 767-773, 2007.

20. Jhawer M, Goel S, Wilson AJ, et al: PIK3CA mutation/PTEN expression status predicts response of colon cancer cells to the epidermal growth factor receptor inhibitor cetuximab. Cancer Res 68: 1953-1961, 2008 (Erratum, Cancer Res 68: 6859, 2008).

21. Padua D, Zhang XH, Wang Q, et al: TGF-beta primes breast tumors for lung metastasis seeding through angiopoietin-like 4 . Cell 133: 66-77, 2008

22. Yanfei $X$ and Boris $P$ : TGF- $\beta$ signaling alterations and susceptibility to colorectal cancer (Review). Hum Mol Genet 1: 14-20, 2007.

23. Markowitz SD and Bertagnolli MM: Molecular origins of cancer: Molecular basis of colorectal cancer. N Engl J Med 361: 2449-2460, 2009.

24. Allinen M, Beroukhim R, Cai L, et al: Molecular characterization of the tumor microenvironment in breast cancer. Cancer Cell 6: 17-32, 2004

25. Frattini M, Saletti P, Romagnani E, et al: PTEN loss of expression predicts cetuximab efficacy in metastatic colorectal cancer patients. Br J Cancer 97: 1139-1145, 2007.

26. Hofer MD, Kuefer R, Varambally S, et al: The role of metastasis-associated protein 1 in prostate cancer progression. Cancer Res 64: 825-829, 2004.

27. Callahan CA, Ofstad T, Horng L, et al: AEMIM/BEG4, a Sonic hedgehog-responsive gene that potentiates Gli-dependent transcription. Genes Dev 18: 2724-2729, 2004.

28. Kimura N, Pilichowska M, Date F, et al: Immunohistochemical expression of somatostatin type $2 \mathrm{~A}$ receptor in neuroendocrine tumors. Clin Cancer Res 5: 3483-3487, 1999.

29. GeneNote/GNF Normal Experimental tissue vectors: twelve normal human tissues (out of 28 tissues shown) hybridized against Affymetrix GeneChips HG-U95A-E and for 22 normal human tissues hybridized against HG-U133A (GNF data**). http://www.genecards.org

30. Kucia M, Reca R, Miekus K, et al: Trafficking of normal stem cells and metastasis of cancer stem cells involve similar mechanisms: pivotal role of the SDF-1-CXCR4 axis. Stem Cells 23: 879-894, 2005

31. Ponomaryov T, Peled A, Petit I, et al: Induction of the chemokine stromal-derived factor-1 following DNA damage improves human stem cell function. J Clin Investig 106: 1331-1339, 2000. 
32. Sun YX, Wang J, Shelburne CE, et al: Expression of CXCR4 and CXCL12 (SDF-1) in human prostate cancers (PCa) in vivo. J Cell Biochem 89: 462-473, 2003.

33. Karkkainen MJ and Petrova TV: Vascular endothelial growth factor receptors in the regulation of angiogenesis and lymphangiogenesis. Oncogene 19: 5598-5605, 2000.

34. Boman BM and Huang E: Human colon cancer stem cells: a new paradigm in gastrointestinal oncology. J Clin Oncol 26: 2828-2838, 2008.

35. O'Brien CA, Pollett A, Gallinger S and Dick JE: A human colon cancer cell capable of initiating tumour growth in immunodeficient mice. Nature 445: 106-110, 2007.

36. Ricci-Vitiani L, Lombardi DG, Pilozzi E, et al: Identification and expansion of human colon-cancer-initiating cells. Nature 445: 111-115, 2007.

37. Dalerba P, Dylla SJ, Park IK, et al: Phenotypic characterization of human colorectal cancer stem cells. Proc Natl Acad Sci USA 104: 10158-10163, 2007.
38. Marhaba R, Klingbeil P, Nuebel T, et al: CD44 and EpCAM: cancer-initiating cell markers (Review). Curr Mol Med 8: 784-804, 2008

39. Liang J, Wan M, Zhang Y, et al: Nanog and Oct4 associate with unique transcriptional repression complexes in embryonic stem cells. Nat Cell Biol 10: 731-739, 2008.

40. Scimone ML, Meisel J and Reddien PW: The Mi-2-like SmedCHD4 gene is required for stem cell differentiation in the planarian Schmidtea mediterranea. Development 137: 1231-1241, 2010.

41. Granovsky M, Fata J, Pawling J, et al: Suppression of tumor growth and metastasis in Mgat5-deficient mice. Nat Med 6: 306-312, 2000.

42. Partridge EA, Le Roy C, Di Guglielmo GM, et al: Regulation of cytokine receptors by Golgi $\mathrm{N}$-glycan processing and endocytosis. Science 306: 120-124, 2004. 